\title{
Treatment of Stage 3 COVID-19 With Transcutaneous Auricular Vagus Nerve Stimulation Drastically Reduces Interleukin-6 Blood Levels: A Report on Two Cases
}

\section{To the Editor:}

The novel coronavirus disease 2019 (COVID-19) progresses in four stages (1). The first stage is a presymptomatic phase, which may lead to stage 2, which presents with fever, cough, generalized malaise, and a high viral load. This is followed, after seven to ten days, by stage 3, which presents with viral pneumonia. Most patients improve clinically as immunological responses are developed at this stage. During stage 3, a small number of patients develop symptoms of hypercytokinemia ("cytokine storm"). Finally, in stage 4, acute respiratory distress syndrome (ARDS) and multiorgan failure sets in, with a high reported mortality rate (1). The respiratory symptoms caused by COVID-19 thus stem from inflammation that causes lung injury. This causes a decrease in oxygen saturation. If the inflammation, marked by the "cytokine storm," is not mitigated, it can lead to ARDS (1); therefore, the aim of therapy should be to abort the "cytokine storm" (2).

Staats et al. (2) reported on two patients who showed marked improvement of clinical symptoms after application of transcutaneous cervical vagus nerve stimulation (VNS), but the authors did not report on objective measurements of at least one of the proinflammatory cytokines. In addition, Jin et al. (3) reported clinical evidence that auricular electroacupuncture, another form of auricular VNS, may inhibit the production of tumor necrotic factor (TNF) alpha, interleukin (IL)-1 beta, IL-6, and IL-8, among others. They argued that it was advantageous for treating the inflammatory processes associated with COVID-19. Finally, there is evidence that IL-6 plasma levels are prognostic indicators of COVID-19 progression $(4,5)$.

We report on two patients with stage 3 COVID-19 in whom IL-6 levels were markedly elevated and in whom transcutaneous auricular VNS (taVNS) may have drastically reduced IL- 6 blood levels over a relatively short period of time. taVNS stimulation was selected on the strength of the Staats et al. (2) and Jin et al. (3) reports. Both patients gave consent to publish these case reports. The institution where the work was done does not require Institutional Review Board approval for single case reports.

\section{CASE PRESENTATIONS}

A 60-year-old male healthcare worker's polymerase chain reaction (PCR) test for Severe Acute Respiratory Syndrome Coronavirus 2 (SARS-CoV-2) nucleic acid was positive. On the day of the test, day 0 , he presented with severe flu-like symptoms with body aches, weakness, and a fever of $99.8^{\circ} \mathrm{F}$. His IL- 6 blood level, taken at 10:00 AM, was $2.7 \mathrm{pg} / \mathrm{mL}$ (reference <6.4). He had no significant preexisting comorbidities other than chronic allergic rhinitis and mild bilateral bronchiectasis. He was treated with azithromycin for three days. The flu-like symptoms resolved the next day, but on day 8 , he presented with severe nonproductive coughing, fever, shortness of breath, and a blood oxygen saturation level of $94 \%$. He also suffered general malaise, and his blood level of IL- 6 , taken before 11:00 AM, was $76.4 \mathrm{pg} / \mathrm{mL}$. Chest x-rays were unremarkable.

The patient received no treatment other than symptomatic paracetamol and codeine to reduce coughing, and he started using taVNS on day 8 as follows: The conchae of both ears were stimulated with a proprietary waveform (Xavant Technology, Silverton, South Africa), with the anode in one ear and the cathode in the other ear. The waveform delivers a 133-kHz decrescendo alternating current in bursts of $0.3 \mathrm{~ms}$ and $25 \mathrm{~Hz}$. The taVNS application was initiated around 8:00 AM every morning and lasted for 60 minutes per day. Blood for IL- 6 blood level measurement was always collected before 11:00 AM. The current was turned up until the patient could just feel it, usually around 4-5 mA. The patient did not receive supplemental oxygen or any other treatment. On day 11 (three days after starting taVNS), although still coughing severely, his saturation returned to $97 \%$, and he generally felt better. On that day (day 11), his IL-6 was $44.1 \mathrm{pg} / \mathrm{mL}$; his C-reactive protein (CRP), which was not measured previously, was $56 \mathrm{mg} / \mathrm{mL}$ (reference <5); his d-dimer was $0.35 \mathrm{mg} / \mathrm{mL}$ (reference 0-0.05); and his procalcitonin was $<0.05 \mathrm{ng} / \mathrm{mL}$ (reference $0-0.05$ ). Treatment with taVNS continued, and on day 14 (five days after starting taVNS), his IL-6 blood levels further declined to $28.0 \mathrm{pg} /$ $\mathrm{mL}$, his CRP was slightly reduced to $55 \mathrm{mg} / \mathrm{mL}$, and he generally felt much better with reduced coughing. His body temperature was normal, and his saturation was between $97 \%$ and $98 \%$. On day 16 (seven days after starting taVNS), he completely recovered from all symptoms; his IL-6 was $7.8 \mathrm{pg} / \mathrm{mL}$, and his CRP was $19 \mathrm{mg} / \mathrm{mL}$. The taVNS treatment was continued for another two days and then discontinued.

Another patient, a 64-year-old man with no preexisting comorbidities, presented with similar symptoms as the above patient, including severe coughing and malaise but abnormal lung $x$-rays and a core temperature of $100^{\circ} \mathrm{F}$. A nasopharyngeal swab for the PCR test for SARS-CoV-2 was negative; however, his clinical

Address correspondence to: André P. Boezaart, MD, PhD, Department of Anesthesiology, University of Florida College of Medicine, 1600 SW Archer Road, PO Box 100254, Gainesville, FL 32610, USA. Email: aboezaart@anest.ufl.edu

Conflict of Interest: The authors reported no conflict of interest.

For more information on author guidelines, an explanation of our peer review process, and conflict of interest informed consent policies, please go to http:// www.wiley.com/WileyCDA/Section/id-301854.html

Source(s) of financial support: None. 
presentation and chest $\mathrm{x}$-rays strongly indicated that he had COVID-19. He had flu-like symptoms seven days prior to presenting to the hospital that subsided the next day (day 0). Upon admission on day 7 , his blood oxygen saturation was $93 \%$, and he received oxygen supplementation. He also received azithromycin, vitamin $A$ and $D$ supplementation, one intravenous injection of $500 \mathrm{mg}$ of methylprednisolone, and routine symptomatic treatment for his fever and cough. Additional treatment was provided with taVNS treatment identical to that of the above patient and initiated on day 7. His progress was similar to that of the patient described above, with blood IL-6 levels of $104.9 \mathrm{pg} / \mathrm{mL}$ and CRP of $264 \mathrm{mg} / \mathrm{mL}$ upon admission on day $7 ; 76.8 \mathrm{pg} / \mathrm{mL}$ and $54 \mathrm{mg} /$ $\mathrm{mL}$, respectively, on day 11 (day 4 of taVNS treatment); and an IL-6 blood value of $36.1 \mathrm{pg} / \mathrm{mL}$ on day 16 . He recovered completely within ten days of admission (day 17), ten days after taVNS was initiated. Although the second patient received methylprednisolone upon admission, in both these patients, our care plan included the initiation of dexamethasone therapy immediately should the patients have shown clinical deterioration or if their IL-6 blood levels had increased or not decreased within 24 hours of starting taVNS. This therapy was not necessary in either of these patients.

\section{DISCUSSION}

The first patient followed the classical presentation through stages 1, 2, and 3 of the disease, while the second patient presented in stage 3 of the disease process when the viral load on the upper respiratory tract was lower or nonexistent. That was most likely the reason he tested negative on his PCR test taken from his nasopharynx. Sputum testing from his lungs may have provided a positive PCR test, but this was not performed. However, upon admission, there was no clinical doubt, supported by his x-ray picture and elevated IL-6 level, that he had stage 3 COVID-19. It is unknown if the steroid injection upon admission was responsible for reducing his IL-6 blood levels and caused him to recover. He did, however, follow the same pattern of recovery as the first patient who did not receive any enteral or parenteral steroids.

Staats et al. (2) proposed two mechanisms by which noninvasive VNS may help patients with respiratory involvement of COVID-19: bronchodilatation and activation of the cholinergic anti-inflammatory pathway (CAP). The CAP upregulates HMGB1, which regulates cytokine expression, leading to balancing of antiand proinflammatory cytokines.

Transcutaneous VNS and taVNS (2) with comparable waveforms have shown positive effects on cytokines, and preliminary work was done in 2020 on their effects on controlling the "cytokine storm" associated with COVID-19 (2). In all of the preliminary experience, no patient has reported adverse effects.
This form of treatment has not yet been proven to be effective in randomized controlled trials (RCTs) to treat the "cytokine storm" associated with SARS-CoV-2 infection. Although we fully appreciate the severe limitations of uncontrolled data, many new treatment modalities started off as single case reports or small case series (2), followed by more specific case reports such as this one, which present more targeted metrics. This may be an example of a specific case report that advances the findings of previous reports and is also based on theoretical principles. Clearly, reports such as these should be followed by RCTs.

We, however, believe that taVNS should be used in addition to a patient's regular care plan because it has no known adverse effects. We propose initial treatment with taVNS therapy and withholding steroid therapy for as long as the IL-6 levels decrease and the patient's condition improves clinically because of the unfavorable side-effect profile of steroids in this scenario. Further scientific evaluation and RCTs are warranted prior to routine use of taVNS with this modality for the treatment of patients with COVID-19.

\section{Authorship Statements}

Drs. Boezaart and Botha both contributed substantially to the study concept and design, acquisition and analysis of data, and drafting and revising of the manuscript. All authors read and approved the final version of this manuscript.

\section{André P. Boezaart MD, PhD, ${ }^{1,2}$ (D; Daniel A. Botha MD, DA(SA),}

${ }^{1}$ The Alon P. Winnie Research Institute, Still Bay, South Africa, and

${ }^{2}$ Division of Acute and Perioperative Pain Medicine, Department of Anesthesiology, University of Florida College of Medicine, Gainesville,

FL, USA

\section{REFERENCES}

1. Girija ASS, Shankar EM, Larsson M. Could SARS-CoV-2-induced hyperinflammation magnify the severity of coronavirus disease (CoViD-19) leading to acute respiratory distress syndrome? Front Immunol 2020;11:1206.

2. Staats P, Giannakopoulos G, Blake J, Liebler E, Levy RM. The use of non-invasive vagus nerve stimulation to treat respiratory symptoms associated with COVID-19: A theoretical hypothesis and early clinical experience. Neuromodulation 2020;23: 784-788. https://doi.org/10.1111/ner.13172.

3. Jin GY, Jin LL, Zheng J, He BJ. Advantages of anti-inflammatory acupuncture in treating sepsis of novel coronavirus pneumonia. World J Tradit Chin Med 2020;6: 188-195.

4. Herold T, Jurinovic V, Arnreich $C$ et al. Elevated levels of IL- 6 and CRP predict the need for mechanical ventilation in COVID-19. J Allergy Clin Immunol 2020;146: 128-136.e4

5. Liu F, Li L, Xu M et al. Prognostic value of interleukin-6, C-reactive protein, and procalcitonin in patients with COVID-19. J Clin Virol 2020;127:104370. 